\title{
Polarization Studies for the eRHIC electron Storage Ring
}

\author{
E. Gianfelice-Wendt*t \\ Fermilab \\ E-mail: elianaefnal.gov
}

\begin{abstract}
A hadron/lepton collider with polarized beams has been under consideration by the scientific community since some years, in the U.S. and Europe. Among the various proposals, those by $\mathrm{JLAB}$ and BNL with polarized electron and proton beams are currently under closer study in the U.S.

In the BNL Ring-Ring design electrons are stored at top energy in a ring to be accommodated in the existing RHIC tunnel. The transversely polarized electron beam is injected into the storage ring at variable top energy, between 5 and $18 \mathrm{GeV}$. Polarization is brought into the longitudinal direction at the IP by a couple of spin rotators. In addition experimenters call for the simultaneous storage of electron bunches with both spin helicities.

In this paper studies of the attainable beam polarization level and lifetime in the storage ring at 18 $\mathrm{GeV}$ are presented.
\end{abstract}

23rd International Spin Physics Symposium - SPIN2018 -

10-14 September, 2018

Ferrara, Italy

\footnotetext{
* Speaker.

${ }^{\dagger}$ This manuscript has been authored by Fermi Research Alliance, LLC under Contract No. DE-AC02-07CH11359 with the U.S. Department of Energy, Office of Science, Office of High Energy Physics.
} 


\section{Introduction}

The realization of a high-energy, high-luminosity polarized Electron-Ion Collider (EIC) has been recognized by the Nuclear Science Advisory Committee (NSAC) in its 2015 Long Range Plan as the highest priority for nuclear science following the completion of the Facility for Rare Isotope Beams. EIC should be able of solving the nucleon spin puzzle and explore the new quantum chromodynamics frontier of ultra-dense gluon fields. Two different EIC designs are under studies in the US, namely eRHIC at the Brookhaven National Laboratory (BNL) and JLEIC at the Thomas Jefferson Laboratory (JLab). Exhaustive descriptions of both designs may be found in [1]. The BNL based EIC design exploits the already existing hadron complex. The schematic layout is shown in Fig. 1.

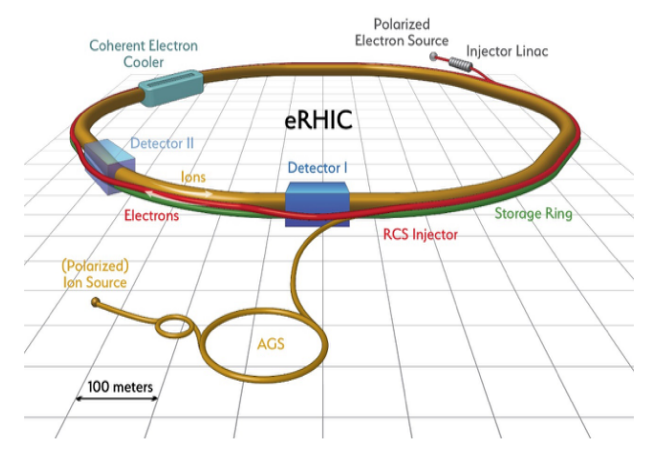

Figure 1: Schematic eRHIC layout.

Because experimenters call for the simultaneous storage of electron bunches with both spin helicities, Sokolov-Ternov effect [2] is not an option for eRHIC as it tends to polarize all bunches in the same direction, namely, for the clockwise rotating electrons, upwards.

Therefore polarized electrons will be generated by a polarized electron source, accelerated in a $400 \mathrm{MeV}$ Linac and in a Rapid Cycling Synchrotron (RCS) to up 5, 12 and $18 \mathrm{GeV}$ and injected at full energy into the electron storage ring. RCS and storage ring will be both accommodated into the $3835 \mathrm{~m}$ long RHIC tunnel. The longitudinal polarization of the electron bunches generated by the source is brought in the vertical direction by a spin rotator prior being injected into the RCS. Single bunches with $\approx 85 \%$ polarization, either up or down, are injected from the RCS into the storage ring where polarization is brought into the longitudinal direction at the Interaction Point (IP) through a couple of solenoidal spin rotators.

\section{Radiative Polarization and eRHIC Storage Ring}

The evolution of beam polarization in electron storage rings is defined by two synchrotron radiation related processes: Sokolov-Ternov self-polarization, and depolarization caused by stochastic photon emission [3]. The self-polarization process leads to a build-up of electron polarization in the direction opposite to the vertical guiding field, up to a maximum of $92.4 \%$ in a perfectly planar storage ring without spin rotators. The polarization time constant decreases as $\gamma^{-5}$. Unless the spin direction is vertical everywhere in phase space, which is the case for a perfectly planar ring without 
spin rotators, stochastic photon emission leads to a randomization of the particle spin directions (spin diffusion) and reduces the equilibrium polarization level.

Polarization builds-up exponentially

$$
P(t)=P_{\infty}\left(1-\mathrm{e}^{-t / \tau_{p}}\right)+P(0) \mathrm{e}^{-t / \tau_{p}}
$$

In the presence of depolarizing effects it is

$$
P_{\infty} \simeq \frac{\tau_{p}}{\tau_{\mathrm{BKS}}} P_{\mathrm{BKS}} \quad \text { and } \quad \frac{1}{\tau_{p}} \simeq \frac{1}{\tau_{\mathrm{BKS}}}+\frac{1}{\tau_{\mathrm{d}}}
$$

where $P_{\mathrm{BKS}}$ and $\tau_{\mathrm{BKS}}$, the Baier-Katkov-Strakhovenko generalization of the Sokolov-Ternov quantities[4], may be computed "analytically", while $\tau_{d}$ and therefore $\tau_{p}$ and $P_{\infty}$, is in general unknown. For eRHIC storage ring at $18 \mathrm{GeV}$ it is $P_{B K S} \simeq 90 \%$ and $\tau_{B K S} \simeq 30$ minutes.

Fig. 2 shows the polarization vs. time under the assumption that the initial polarization is $\pm 85 \%$ and the asymptotic polarization is $+30 \%$. After 5 minutes the polarization of upwards polarized bunches is still $60 \%$ and the 5 minutes average is $73 \%$, while for downwards polarized bunches the polarization decays to $-39 \%$ and the average is only $-61 \%$.

Fig. 3 shows polarization after 5 minutes (red) and the 5 minutes average (blue) vs. asymptotic polarization for $P(0)=-85 \%$.

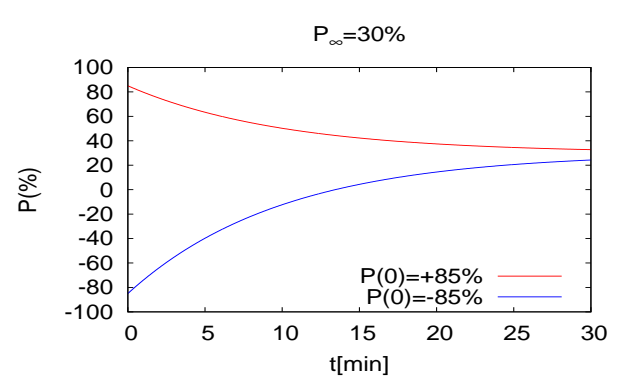

Figure 2: $P$ vs. time for $P(0)= \pm 85 \%$ and $P_{\infty}=30 \%$.

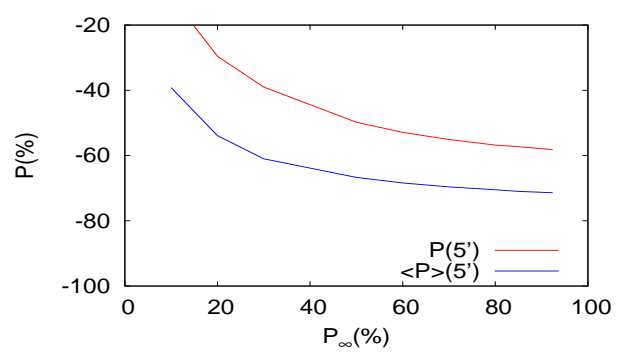

Figure 3: $P$ after 5 minutes (red) and the 5 minutes average (blue) vs. asymptotic polarization for $P(0)=-85 \%$. 
We see that above $P_{\infty} \simeq 50 \%$ the absolute value of the average increases only very slowly with $P_{\infty}$. Achieving $-70 \%$ average polarization on 5 minutes would require an extremely challenging $80 \%$ asymptotic polarization. With $P_{\infty}=50 \%$ the downwards polarized bunches would need to be replaced every 4 minutes for keeping the average polarization at $-70 \%$.

\section{Simulations for the eRHIC storage ring}

\subsection{Polarization in the unperturbed ring}

Figure 4 shows polarization in linear approximation for the unperturbed machine with luminosity tunes $Q_{x}=60.12, Q_{y}=56.10$ and $Q_{s}=0.046$ and in presence of one pair of spin rotators, vs. $a \gamma$ ( $a$ electron gyromagnetic anomaly), which is the spin tune for a planar machine w/o solenoids. In Figure 4, as well as in the following Figs. 7, 10 and 11, the red, blue and magenta lines refer to the polarization related to the radial, vertical and longitudinal motion respectively. During the energy scan the settings of the solenoid are fixed; however $\delta \hat{n}_{0}$, the deviation of the periodic solution to the Thomas-BMT equation [6][7] from the ideal one, is relatively small over the whole energy range (see Fig. 5).

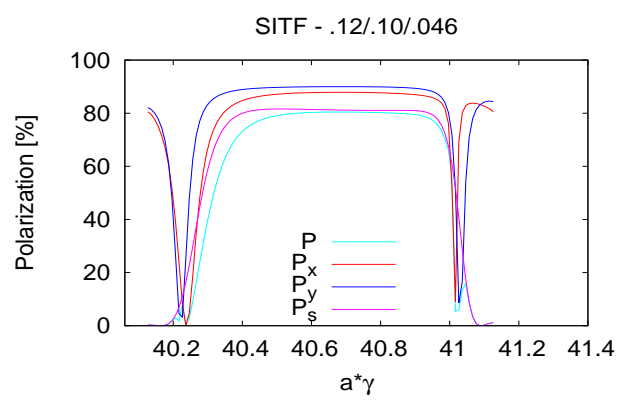

Figure 4: Linear polarization vs. a $\gamma$ for the unperturbed lattice as computed by SITF [5].

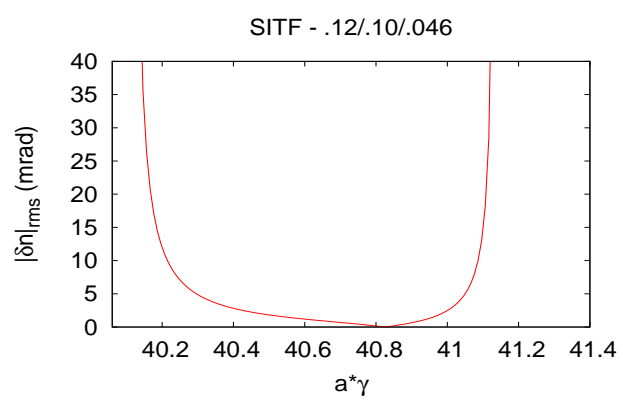

Figure 5: RMS value of $\delta \hat{n}_{0}$ vs. $a \gamma$ for the unperturbed lattice (SITF).

Figure 6 shows polarization as computed by the tracking code SITROS [5]. It can be expected that, manifesting the presence of higher order resonances and synchrotron side-bands, tracking 
calculations give lower polarization than calculations with linearized spin motion. However the discrepancy seems exceedingly large in this case. This may be related to the fact that the vertical emittance as computed by the tracking is about 8 times larger than expected or to the fact that the IR optics is not fully spin matched.

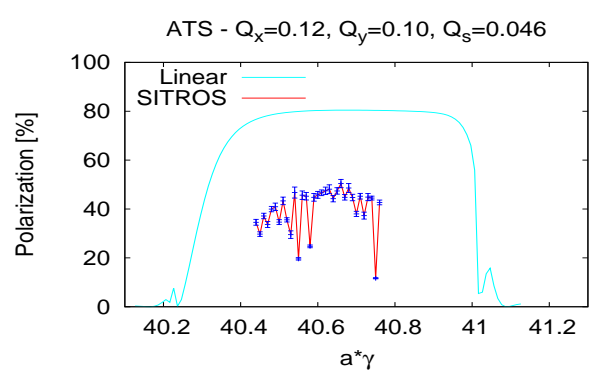

Figure 6: Polarization vs. $a \gamma$ for the unperturbed lattice from SITROS tracking. The cyan line is the linear polarization.

\subsection{Polarization in presence of misalignments}

Gaussian distributed random misalignments have been added to all 494 quadrupoles, with RMS value $\delta x=\delta y=200 \mu \mathrm{m}$, for the horizontal and vertical misalignment, and $\delta \Psi=200 \mu \mathrm{rad}$ for the roll angle. To each quadrupole one Beam Position Monitor (BPM), measuring beam position in both planes, and one horizontal an one vertical corrector have been added.

It turns out that, due to the large coupling in the solenoid sections, the orbit can't be corrected in a satisfactory manner in the two planes separately in the whole machine as done by the MAD-X correction module. Therefore the two planes are corrected simultaneously with an "external" code and corrections are read back by MAD-X.

Polarization after correcting the closed orbit down to $x_{r m s} \simeq 137 \mu \mathrm{m}$ and $y_{r m s} \simeq 71 \mu \mathrm{m}^{1}$ is shown in Figure 7 for one error realization. Figure 8 shows the corresponding polarization axis tilt.

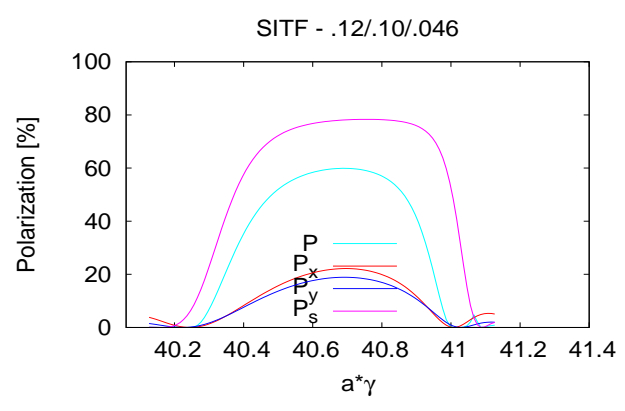

Figure 7: Linear polarization vs. $a \gamma$ for the perturbed lattice after closed orbit correction (SITF).

\footnotetext{
${ }^{1}$ Contribution from synchrotron radiation is included.
} 


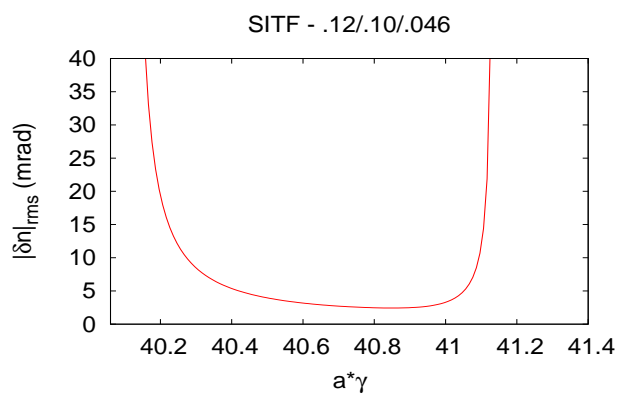

Figure 8: RMS value of $\delta \hat{n}_{0}$ vs. $a \gamma$ for the perturbed lattice after closed orbit correction (SITF).

Although the closed orbit is well corrected and $\delta \hat{n}_{0}$ is small, $P_{x}$ and $P_{y}$ are little. The fact that polarization (the cyan line) is larger than $P_{x}$ and $P_{y}$ is due to an interference which does not take place when the spin motion is not linearized (see Figure 9).

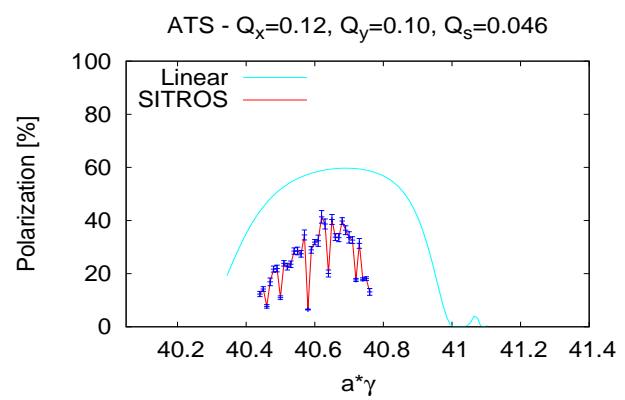

Figure 9: Polarization vs. $a \gamma$ for the perturbed lattice after closed orbit correction (SITROS tracking). The cyan line is the linear polarization.

The reason of the little polarization is the large betatron coupling, $\left|C^{-}\right| \simeq 0.01 .46$ independently powered skew quadrupoles in arc locations where $D_{x} \sqrt{\beta_{y}}$ and $\sqrt{\beta_{x} \beta_{y}}$ are large, are used to correct linear coupling[8] and spurious vertical dispersion simultaneously. The resulting linear polarization is shown in Figure 10.

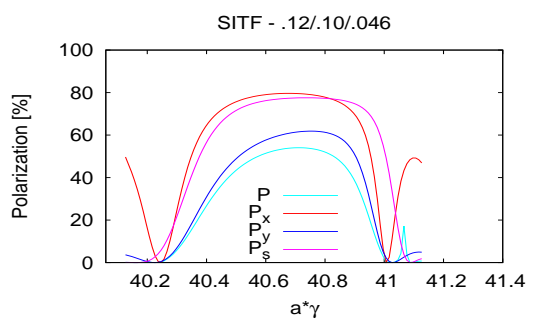

Figure 10: Linear polarization vs. $a \gamma$ for the perturbed lattice after closed orbit, spurious vertical dispersion and betatron coupling correction.

Polarization is further improved by correcting in addition $\delta \hat{n}_{0}$ by harmonic bumps [9] (see 
Figures 11 and 12).

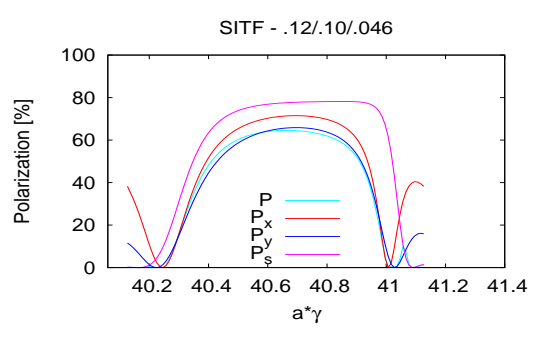

Figure 11: Linear polarization vs. $a \gamma$ for the perturbed lattice after closed orbit, spurious vertical dispersion, betatron coupling and $\delta \hat{n}_{0}$ correction.

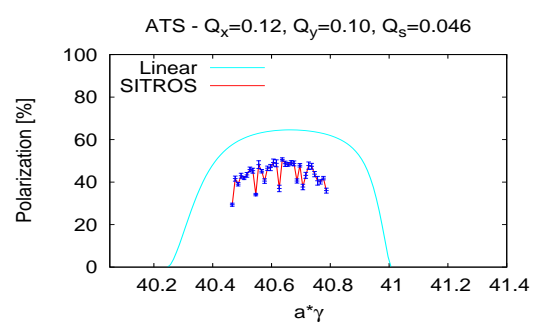

Figure 12: Polarization vs. $a \gamma$ for the perturbed lattice after closed orbit, spurious vertical dispersion, betatron coupling and $\delta \hat{n}_{0}$ correction (SITROS tracking). The cyan line is the linear polarization.

With all corrections in place the level of polarization from tracking is the same as for the unperturbed machine (compare with Figure 6).

\section{Summary}

The impact of Sokolov-Ternov effect on the beam polarization in the eRHIC storage ring at $18 \mathrm{GeV}$ has been assessed and results for the expected asymptotic polarization in presence of quadrupole misalignments for one particular error realization has been shown. By carefully correcting closed orbit, betatron coupling, spurious vertical dispersion and polarization axis distortion it is possible to recover the polarization level of the unperturbed machine, namely about 50\%. Assuming that the starting polarization is $\pm 85 \%$, the downwards polarized bunches would need to be replaced each 4 minutes for achieving an average polarization of $75 \%$. The electron beam vertical size at the IP after correction is $4.4 \mu \mathrm{m}$. The mismatch with the larger proton beam size will likely require to blow up the electron beam size at the IP, without sacrificing polarization. This together with the investigation of the effect of beam-beam on the electron polarization are the most urgent issues to be addressed.

\section{References}

[1] Y. H. Chin, E. Gianfelice-Wendt (eds.), Beam Dynamics Newsletter No.74, August 2018. 
[2] A. A. Sokolov, I. M. Ternov, "On Polarization and spin effects in the theory of synchrotron radiation", Sov.Phys.Dokl., vol. 8, pp. 1203-1205 (1964).

[3] Y. S. Derbenev, A. M. Kondratenko, "Diffusion of particle spins in storage elements", Sov. Phys. JETP, 35:230 (1972).

[4] V. N. Baier, V. M. Katkov, V. M. Strakhovenko, "Kinetics of Radiative Polarization", Sov.Phys.JETP., vol. 31, pp. 908-911 (1970).

[5] J. Kewisch, "Depolarisation der Elektronenspins in Speicherringen durch nichtlineare Spin-Bahn-Kopplung", Ph.D. thesis, Phys.Dept., Hamburg Univ. , Hamburg, Germany, 1985.

[6] L. H. Thomas, "The Kinematics of an electron with an axis”, Phil. Mag., vol. 3, pp.1-21 (1927).

[7] V. Bargmann, L. Michel and V. L. Telegdi, "Precession of the polarization of particles moving in a homogeneous electromagnetic field”, Phys. Rev. Lett., vol. 2, pp. 435-436 (1959).

[8] Y. Alexahin, E. Gianfelice-Wendt, "A new algorithm for the correction of the linear coupling at Tevatron", presented at EPAC’06, Edinburgh, U.K., June 2006, paper WEPCH055, pp. 2047-2049.

[9] D. P. Barber et al.,"High spin polarization at the HERA Electron Storage Ring",Nucl. Instrum. Meth., vol. A338, pp. 166-184 (1994). 